\title{
ATP Precursor Depletion and Postischemic Myocardial Recovery ${ }^{1}$
}

\author{
S'teven F. Bolling, M.D., ${ }^{2}$ Edward L. Bove, M.D., and Kim P. Gallagher, Ph.D. \\ Thoracic Surgery Research Laboratory, Departments of Surgery (Section of Thoracic Surgery) and Physiology, \\ University of Michigan Medical School, Ann Arbor, Michigan 48109
}

Presented at the Annual Meeting of the Association for Academic Surgery, Houston, Texas, November 14-17, 1990

\begin{abstract}
Although cardioplegia reduces myocardial metabolism during ischemia, adenosine triphosphate (ATP) depletion occurs, which may contribute to poor functional recovery after reperfusion. Augmenting myocardial adenosine during ischemia is successful in improving ATP repletion and myocardial recovery following ischemia. If adenosine is an important determinant of ischemic tolerance, then depletion or elimination of myocardial adenosine should lead to poor functional and metabolic recovery after ischemia. To test this hypothesis, isolated, perfused rabbit hearts were subjected to $120 \mathrm{~min}$ of $34^{\circ} \mathrm{C}$ ischemia. Hearts received St. Thomas cardioplegia alone or cardioplegia containing $200 \mu M$ adenosine, or cardioplegia containing 15,5 , 2.5 , or $0.025 \mu \mathrm{g} / \mathrm{ml}$ adenosine deaminase (ADA), which catalyzes the breakdown of adenosine to inosine, making adenosine unavailable as an ATP precursor. Functional recovery was determined and myocardial nucleotide levels were measured before, during, and after ischemia. Following ischemia and reperfusion, control hearts recovered to $51 \pm 3 \%$ of preischemic developed pressure (DP). There was significantly better recovery in adenosine-augmented hearts $(68 \pm 7 \%)$, while ADA hearts had significantly worse recovery. Hearts treated with $0.025 \mu \mathrm{g} / \mathrm{ml}$ ADA recovered to only 29 $\pm 5 \%$ of DP and higher dose ADA hearts failed to demonstrate any recovery of systolic function. Furthermore, adenosine enhanced metabolic recovery, whereas ADA resulted in greatly depleted ATP and precursor reserves. Postischemic developed pressure closely paralleled the availability of myocardial adenosine, consistent with the hypothesis that myocardial adenosine levels at end ischemia and early reperfusion are important determinants of functional recovery after global ischemia. (c) 1991 Academic Press, Inc.
\end{abstract}

\section{INTRODUCTION}

To provide myocardial protection during cardiac surgery, cardioplegia and myocardial hypothermia are uti-

\footnotetext{
${ }^{1}$ Supported in part by NIH Grant R01 HL32043; and by the American Heart Association of Michigan.

${ }^{2}$ To whom reprint requests should be addressed at the University of Michigan Hospital, Section of 'Thoracic Surgery, 2120D Taubman Center, Box 0344, Ann Arbor, MI 48109.
}

lized routinely. Despite these protective techniques, however, adenosine triphosphate (ATP) depletion may occur during ischemia. The slow recovery of the depleted nucleotides, especially ATP, following ischemia is well recognized. Since intracellular ATP is essential for myocardial contraction and relaxation, ATP depletion may be an important factor contributing to the incomplete recovery of ventricular function following ischemia and reperfusion. The decrease in ATP during ischemia is not accompanied by a rise in ADP, as ADP is quickly transformed to AMP. The dephosphorylation of AMP by $5^{\prime}$-nucleotidase results in formulation of adenosine which is deaminated to inosine by adenosine deaminase. Since the nucleosides can exit the myocyte, they are washed out of the interstitial space during reflow and are unavailable for the nucleotide salvage pathway.

Previous studies [1-5] have demonstrated that adenosine-supplemented cardioplegia was associated with accelerated repletion of ATP and improved functional recovery after reperfusion. Two-deoxycoformycin, an agent that inhibits adenosine deaminase, was also shown to be beneficial [3], supporting the view that adenosine availability during and after global ischemia may be an important determinant of postischemic metabolic and functional recovery. If this hypothesis is correct, then depletion or elimination of myocardial adenosine should lead to poor functional and metabolic recovery after ischemia.

Cardioplegia containing adenosine deaminase was administered to isolated, perfused rabbit hearts subjected to global ischemia. Adenosine deaminase was used to accelerate the breakdown of adenosine, limiting its availability as an ATP precursor. The effects of adenosine depletion on functional and metabolic recovery were compared with the results obtained when adenosine levels were augmented and with a control group of isolated hearts treated with cardioplegia alone.

\section{MATERIAL AND METHODS}

Studies were performed in isolated, perfused rabbit hearts. Male New Zealand White rabbits ( 3 to $4 \mathrm{~kg}$ ) were anesthetized and cardiectomy was performed. The beating heart was suspended from a perfusion column by aortic cannulation and coronary perfusion was estab- 
lished at $80 \mathrm{~mm} \mathrm{Hg}$. The perfusate used was a modified, oxygenated Krebs-Ringer bicarbonate solution ( $\mathrm{pH} 7.44$ to $7.48,37^{\circ} \mathrm{C}, 300$ to $310 \mathrm{mOsm} /$ liter), with an oxygen tension of 550 and 650 torr. The perfusion solution was filtered and was not recirculated.

While the heart was perfused on the column, a portion of the mitral valve with its chordae was excised and a latex balloon, connected to saline-filled tubing, was introduced into the left ventricle (LV) through the mitral orifice. The balloon was sewn in place in such a way to allow for passive venting of the LV cavity. The balloon was connected through a catheter to a pressure transducer, and LV pressure was recorded continuously. Output from the pressure transducer was electronically differentiated to enable continuous recording of $d P / d t$.

During the preischemic control period, a volume of saline was introduced into the $\mathrm{LV}$ balloon to produce an end-diastolic pressure (EDP) of $10 \mathrm{~mm} \mathrm{Hg}$. The same volume was used to evaluate ventricular systolic performance during reperfusion. Coronary flow was measured volumetrically. A thermistor needle was inserted into the midmyocardium to record myocardial temperature.

After a 30-min stabilization period, control measurements of end-diastolic pressure (EDP), developed pressure (DP, peak systolic pressure minus end-diastolic pressure), peak positive $d P / d t$, and coronary flow were made in each heart. Hearts were then rendered globally ischemic. The intraventricular balloon was deflated and $60 \mathrm{ml}$ of cardioplegia was administered. All hearts were maintained at $34^{\circ} \mathrm{C}$ by means of a circulating water jacket during $120 \mathrm{~min}$ of total ischemia. All hearts received $15 \mathrm{ml}$ of cardioplegia every $30 \mathrm{~min}$ during ischemia. Control hearts $(n=9)$ received modified St. Thomas cardioplegia alone, while experimental hearts received modified St. Thomas cardioplegia plus $200 \mu M$ adenosine $(n=9)$ or adenosine deaminase $15,5,2.5$, or $0.025 \mu \mathrm{g} / \mathrm{ml}$ of cardioplegia $(n=5,5,5$, and 4 , respectively).

Reperfusion was performed with perfusate temperature at $37^{\circ} \mathrm{C}$ and delivered with pressure at $80 \mathrm{~mm} \mathrm{Hg}$. Defibrillation was performed as needed. During the initial $15 \mathrm{~min}$ of reperfusion, the intraventricular balloon was kept deflated to simulate the beating, nonworking condition. After the initial $15 \mathrm{~min}$ of reperfusion, the LV balloon was refilled to the preischemic control volume and measurements of EDP, DP, $d P / d t$, and coronary flow were made. The balloon remained inflated for the remainder of reperfusion. $\mathrm{LV}$ functional measurements were obtained after 15,30 , and $45 \mathrm{~min}$ of reperfusion. After $45 \mathrm{~min}$ of reperfusion, all hearts were removed from the perfusion apparatus. This experimental design for this model is well established and has been published previously $[3,4]$.

A parallel series of experiments were done concurrently in an identical fashion to determine myocardial nucleotide levels. Control hearts $(n=4)$ received modified St. Thomas cardioplegia, while experimental hearts received modified St. Thomas cardioplegia with the addition of $200 \mu \mathrm{M}$ adenosine $(n=4)$ or adenosine deaminase $15,5,2.5$, or $0.025 \mu \mathrm{g} / \mathrm{ml}$ of cardioplegia $(n=4$ each). Specimens were taken from the left ventricle with a biopsy gun during the control period, prior to reflow (at end ischemia), and 1 and 15 min during reperfusion. The biopsy specimens were frozen in liquid nitrogen immediately after they were obtained.

The specimens were assayed by the high performance liquid chromatography (HPLC) method for myocardial cellular ATP, ADP, AMP, adenosine, and phosphocreatine levels and values are expressed as micromoles of nucleotide per milligram of protein. Statistical analysis was performed with analysis of variance and Student's two-tailed $t$ test, where appropriate. A $P$ value of less than 0.05 was considered significant. All results are expressed as means \pm SEM. All animals received humane care in compliance with the Principles of Laboratory Animal Care formulated by the National Society for Medical Research.

\section{RESULTS}

\section{Left Ventricular Function}

There were no significant differences in the prearrest $\mathrm{DP}, \mathrm{dP} / \mathrm{dt}$, or heart rates between control hearts and those receiving adenosine or adenosine deaminase for any group. Left ventricular systolic functional measurements of DP and $+\mathrm{dP} / \mathrm{dt}$ were made after 15,30 , and 45 min of reperfusion. Left ventricular EDP was also measured in 15-min intervals following reperfusion and was expressed as an increase in EDP over the preischemic control EDP value of $10 \mathrm{~mm} \mathrm{Hg}$.

After $120 \mathrm{~min}$ of hypothermic ischemia and $45 \mathrm{~min}$ of reperfusion control hearts recovered to $51 \pm 3 \%$ of the preischemic DP. There was significantly better recovery of $\mathrm{LV}$ function in the adenosine-augmented hearts with $68 \pm 7 \%$ recovery of DP in the hearts treated with 200 $\mu M$ exogenous adenosine ( $P<0.05$ vs control hearts). However, the adenosine deaminase (ADA)-treated hearts had significantly worse recovery of function than those of the control hearts. Hearts treated with 0.025 $\mu \mathrm{g} / \mathrm{ml} \mathrm{AD \Lambda}$ recovered to only $29 \pm 5 \%$ of preischemic DP and higher dose ADA hearts failed to demonstrate any recovery of systolic function (Table 1).

Left ventricular EDP, an estimate of diastolic function in this model, was significantly lower at all times during reperfusion in the adenosine-treated (ADO)-augmented hearts compared with control. After $45 \mathrm{~min}$ of reperfusion, control hearts demonstrated elevated left ventricular end-diastolic pressures with a $24 \pm 4 \mathrm{~mm} \mathrm{Hg}$ rise in LVEDP, whereas the $200 \mu M$ adenosine hearts had only a $16 \pm 4 \mathrm{~mm} \mathrm{Hg}$ elevation in LVEDP. The ADA hearts displayed a $40 \pm 8 \mathrm{~mm} \mathrm{Hg}, 39 \pm 7 \mathrm{~mm} \mathrm{Hg}, 52 \pm 6$ $\mathrm{mm} \mathrm{Hg}$, and $59 \pm 2 \mathrm{~mm} \mathrm{Hg}$ rise in LVEDP, with ADA 
TABLE 1

Functional Recovery 45 Min following Reperfusion

\begin{tabular}{lcccc}
\hline & $\mathrm{N}$ & \%DP & dEDP & CF \\
\hline ADO & 9 & $68 \pm 7^{*}$ & $16 \pm 4^{*}$ & $38 \pm 7$ \\
$\mathrm{CTL}$ & 9 & $51 \pm 3$ & $24 \pm 4$ & $36 \pm 4$ \\
$\mathrm{ADA}-0.025 \mu \mathrm{g} / \mathrm{ml}$ & 4 & $29 \pm 5^{*}$ & $40 \pm 8^{*}$ & $35 \pm 7$ \\
$\mathrm{ADA}-2.5 \mu \mathrm{g} / \mathrm{ml}$ & 5 & $0^{*}$ & $39 \pm 7^{*}$ & $31 \pm 6$ \\
$\mathrm{ADA}-5 \mu \mathrm{g} / \mathrm{ml}$ & 5 & $0^{*}$ & $52 \pm 6^{*}$ & $40 \pm 6$ \\
$\mathrm{ADA}-15 \mu \mathrm{g} / \mathrm{ml}$ & 5 & $0^{*}$ & $59 \pm 2^{*}$ & $31 \pm 5$ \\
\hline
\end{tabular}

Note. ADO, adenosine treated hearts; CTL, control hearts; ADA, hearts treated with adenosine deaminase; $\% \mathrm{DP}$, recovery of developed pressure presented as a percentage return of preischemic developed pressure; dEDP, change in end diastolic pressure following reperfusion as compared to preischemic baseline isovolumic end diastolic pressure; CF, coronary flow.

${ }^{*} P<0.05$ vs controls.

doses of $0.025 \mu \mathrm{g} / \mathrm{ml}, 2.5 \mu \mathrm{g} / \mathrm{ml}, 5 \mu \mathrm{g} / \mathrm{ml}$, and $15 \mu \mathrm{g} / \mathrm{ml}$, respectively, in the cardioplegia (Table 1).

Coronary flow during reperfusion demonstrated no differences among any of the groups. Preischemic coronary flow values were also similar in all of the groups. Finally, heart rates demonstrated no significant differences among any of the groups following reperfusion.

\section{Nucleotide Levels}

Adenosine, AMP, ADP, and ATP were measured in the control, adenosine, and adenosine deaminase groups ( $n=4$ each) before, during, and following ischemia. Results are expressed as $\mu M$ of nucleotide per milligram of protein (measured by Lowry assay) and are summarized in Table 2. Baseline readings of adenosine $(2.4 \pm 0.3$ $\mu M / \mathrm{mg}$ protein) and ATP (2.6 $\pm 0.8 \mu M / \mathrm{mg}$ protein) were equivalent in all groups.

During ischemia, ATP levels decreased in all groups. The end-ischemic ATP level was reduced to approximately $0.8 \mu \mathrm{M} / \mathrm{mg}$ protein in the control, adenosineaugmented, and low dose ADA groups, while ATP was essentially eliminated in the high dose ADA groups. Also during ischemia, myocardial adenosine was depressed in the control and low dose ADA groups, increased in the ADO-treated group, and reduced to zero in the high dose ADA groups during ischemia. Control hearts were characterized by adenosine levels of $2.0 \pm 0.12 \mu \mathrm{M} / \mathrm{mg}$ at end ischemia; the values were $5.6 \pm 0.17$ in the adenosinetreated group ( $P<0.05$ vs control). Hearts treated with ADA had no measurable adenosine except in the 0.025 $\mu \mathrm{g} / \mathrm{ml}$ ADA group, which had adenosine levels of 1.6 $\pm 0.03 \mu \mathrm{M} / \mathrm{mg}$ at end ischemia.

During reperfusion, both at 1 and $15 \mathrm{~min}$ following reflow, control hearts remained characterized by depressed adenosine levels $(1.9 \pm 0.5$ and $1.5 \pm 0.07 \mu \mathrm{M} / \mathrm{mg}$ protein). ATP levels also remained low in the control group with values of only $1.8 \pm 0.05$ and $1.3 \pm 0.19 \mu \mathrm{M} /$ $\mathrm{mg}$ at 1 and $15 \mathrm{~min}$ following reperfusion, respectively.

In comparison, adenosine-treated hearts had high postischemic adenosine levels $(5.9 \pm 0.09$ and $4.0 \pm 0.11$ $\mu M / \mathrm{mg}, 1$ and $15 \mathrm{~min}$ following reflow). The adenosinetreated hearts also had substantially higher levels of ATP at 1 and 15 min following reflow with levels of 5.9 \pm 0.05 and $6.0 \pm 0.07 \mu M / \mathrm{mg}$, respectively $(P<0.05$ vs control).

Low dose ADA $(0.025 \mu \mathrm{g} / \mathrm{ml})$-treated hearts had adenosine levels of $1.8 \pm 0.02$ and $0 \mu M / \mathrm{mg}$ at 1 and 15 min following reperfusion. Furthermore, this lowest dose ADA-lrealed group $(0.025 \mu \mathrm{g} / \mathrm{ml})$ was characterized by depressed levels of ATP immediately after reperfusion and elimination of ATP 15 min postreperfusion. Consistent with the experimental hypothesis, higher dose ADA-treated $(2.5,5$, and $15 \mu \mathrm{g} / \mathrm{ml}$ adenosine deaminase) hearts displayed complete depletion of adenosine and also ATP during and after ischemia.

\section{DISCUSSION}

In the present study, postischemic developed pressure closely paralleled the availability of myocardial adenosine (Fig. 1), consistent with the hypothesis that myo-

TABLE 2

Metabolic Recovery 45 Min following Reperfusion

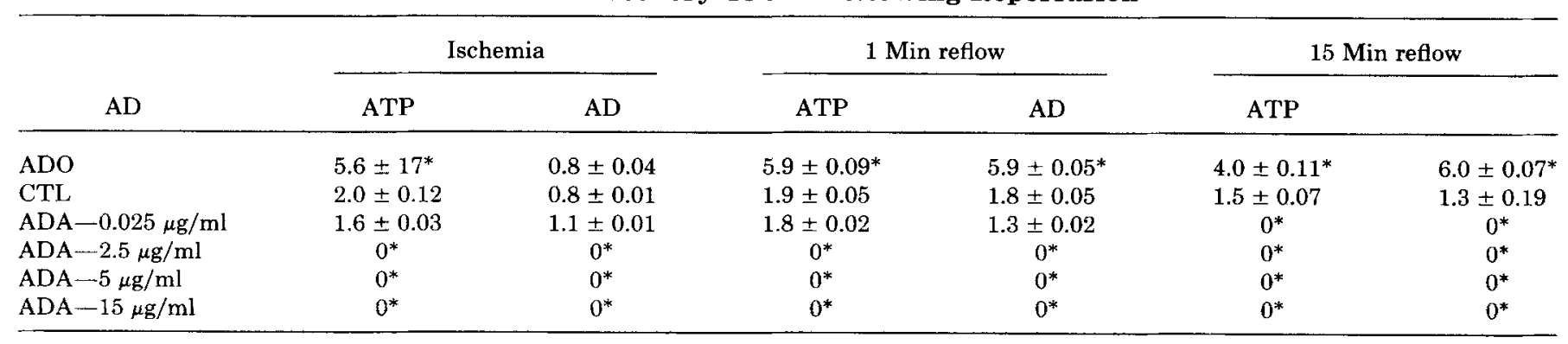

Note. ADO, adenosine-treated hearts; CTL, control hearts; ADA, adenosine deaminase-treated hearts; nucleotide lcvels are presented as $\mu M$ of nucleotide/mg of protein; $\mathrm{AD}$, nucleotide levels of adenosine; ATP, nucleotide levels of adenosine triphosphate.

${ }^{*} P<0.05$ vs controls. 


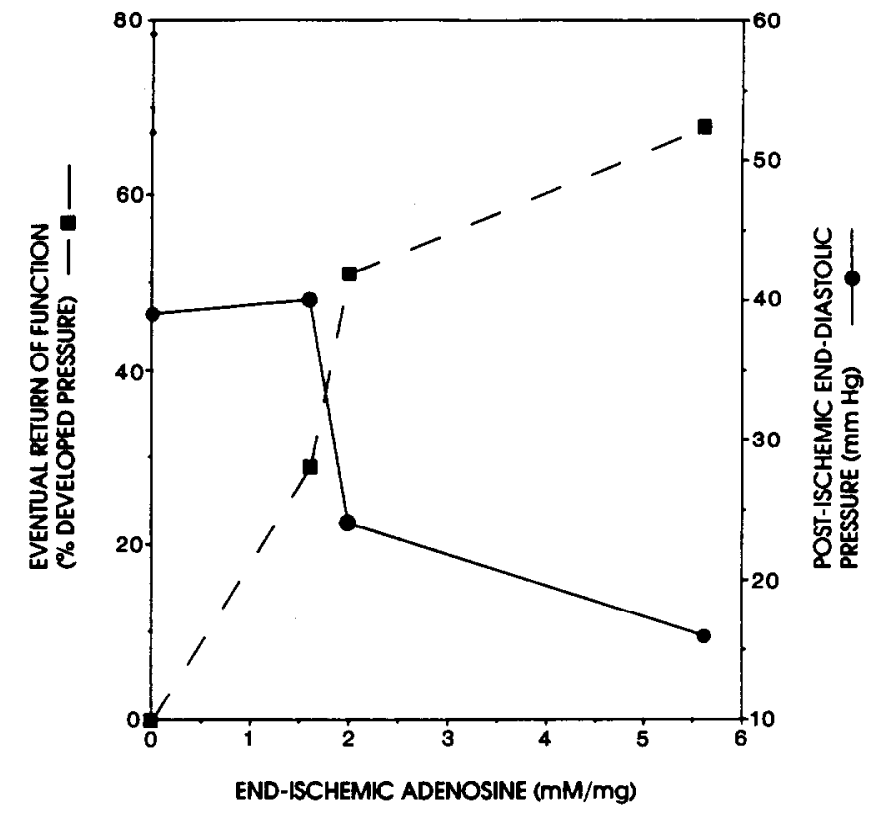

FIG. 1. Correlation of end-ischemic adenosine vs eventual return of $\mathrm{LV}$ function and postischemic end diastolic pressure ( $45 \mathrm{~min}$ postreperfusion).

cardial adenosine levels at end ischemia and early reperfusion are important determinants of functional recovery. ATP depletion occurred during ischemia; however, ATP content did not predict myocardial recovery following ischemia. Additionally, postischemic end-diastolic pressure was inversely correlated to myocardial adenosine levels, not ATP at end ischemia (Fig. 1). In accordance with our hypothesis relating available adenosine to systolic and diastolic functional recovery, ATP resynthesizing capacity after an ischemic episode was depressed or abolished when adenosine deaminase was administered exogenously in cardioplegia. We speculate that the mechanism of adenosine deaminase's action in this model is mediated through decreased availability of intracellular adenosine at reperfusion, thereby eliminating or greatly altering the ability of the myocardium to resynthesize ATP. While intracellular adenosine is difficult to demonstrate, myocardial levels of adenosine, as shown by HPLC from biopsies, were shown to correlate well with functional recovery. Accordingly, functional recovery was poor or nonexistent when adenosine reserves were manipulated and depleted with adenosine deaminase.

In previous studies, it was demonstrated that nucleotide levels decrease during ischemia [1-5]. Since ATP is essential for myocardial contraction and relaxation, any depletion of ATP, such as that seen during ischemia, may have a detrimental effect upon postischemic myocardial functional recovery. Reduction in myocardial ATP content has been correlated with the incomplete recovery of ventricular function observed following ischemia [1].
Other investigations have demonstrated a favorable effect of ATP precursor enhancement on the recovery of ventricular function following ischemia [5, 6]. In a previous study from this laboratory, for example [4], a beneficial effect of the exogenous adenosine on recovery of ventricular function was demonstrated in a isolated adult rabbit heart model. In that study, control hearts recovered $47 \pm 3 \%$ of their initial developed pressure following $2 \mathrm{hr}$ of hypothermic $\left(32^{\circ} \mathrm{C}\right)$ multidose cardioplegic-induced ischemia and $45 \mathrm{~min}$ of reperfusion. Hearts receiving cardioplegia supplemented with 100 , 200 , or $400 \mu M$ adenosine had significantly increased recovery of function $(63 \pm 4 \%, 78 \pm 3 \%$, and $70 \pm 4 \%$ of initial developed pressure, respectively).

In a follow-up study [3] we determined if augmentation of myocardial adenosine during global ischemia improves functional recovery by reducing depletion of ATP during ischemia or by enhancing repletion of ATP after reperfusion. Isolated adult rabbit hearts underwent 120 min of mildly hypothermic ischemia $\left(34^{\circ} \mathrm{C}\right)$. This study demonstrated that adenosine augmentation with exogenous adenosine or with 2-deoxycoformycin (a noncompetitive inhibitor of adenosine deaminase) or the combination of these agents significantly enhanced recovery of ventricular function following ischemia, as compared to standard cardioplegia-treated controls. Nucleotide levels in tissue biopsies were also measured by HPLC. During ischemia, adenosine levels were significantly elevated in the adenosine-augmented groups, but not in the control group. During ischemia, ATP decreased equally in all groups, indicating that augmenting myocardial adenosine had no effect on depletion of ATP during ischemia.

After reperfusion, ATP levels remained depressed in the control group, but increased in the adenosine-augmented groups above baseline values, suggesting that the improved functional recovery noted was due to accelerated repletion and resynthesis of adenine nucleotide stores in the adenosine-augmented groups.

Adenine nucleotides are resynthesized following ischemia by two major pathways [7]. The first pathway is by de novo synthesis of the nucleotide pool. However, this pathway is extremely slow and energy consuming [8]. The second or salvage pathway involves rephosphorylation of adenosine to AMP by the enzyme adenosine kinase and subsequent regeneration of ATP. The salvage pathway is efficient and rapid, but it depends on the availability of intracellular adenosine as a precursor for ATP resynthesis. When there is depletion of intracellular adenosine by degradation to inosine (catalyzed by adenosine deaminase) and washout of the nucleosides at reflow, then precursors are not available for the salvage pathway and resynthesis of ATP. We demonstrated in our previous study [3] that modification of adenosine deaminase activity can be an important determinant of intracellular nucleotide status and, potentially, recovery of postischemic function, because inhibition of adeno- 
sine deaminase during ischemia with 2-deoxycoformycin was beneficial in terms of functional recovery.

The uptake and salvage of adenosine and its breakdown product inosine has been identified in isolated mature cardiac myocytes [9] by measuring the distribution of radioactive precursor nucleotides incorporated into ATP, ADP, and AMP. When the maximum rates of the reactions in the nucleotide salvage pathway were calculated, it was noted that myocytes have little capacity to salvage inosine. Additionally, inosine leaves the myocyte much faster than adenosine and by a passive mechanism. Adenosine is the favored substrate for the nucleotide salvage pathway, but the rate of adenosine salvage for intracellular adenosine is eight times the rate for extracellular adenosine used as substrate for the salvage pathway.

Adenosine deaminase is involved in many physiologic interactions, including the control and local regulation of hyperemic vasodilation, in many vascular beds [10, 11]. However, coronary flow during reperfusion demonstrated no differences among any of the control or treated groups, probably secondary to the maximal vasodilation seen at all times in this crystalloid perfused model. Since coronary flow was not altered by the manipulations in this study, the mechanism of adenosine's relationship to the return of postischemic function must be based on other biologic effects of adenosine. Alteration in the physiologic levels of adenosine deaminase can have many profound effects and lead to a number of disease states [12-14]. The enzyme adenosine deaminase is present in all myocytes and endothelial cells found in the heart. The equilibrium constant for adenosine deaminase strongly favors the formation of inosine from adenosine. Furthermore, due to the relatively high $V_{\max }$ of the enzyme adenosine, at high concentrations of intracellular adenosine in the myocytes, adenosine deaminase is probably the major pathway for degradation of adenosine [15].

In the present study, exogenously administered adenosine deaminase was used to eliminate or reduce myocardial adenosine in order to determine the consequences of adenosine depletion on myocardial functional recovery. This study reinforces the concept that adenosine is an important determinant of ischemic tolerance, as depletion or elimination of myocardial adenosine by adenosine deaminase leads to poor functional and metabolic recovery after ischemia. Enhancing purine precursor profile during the time of ischemia may represent an important potential strategy in cardiac surgery.

\section{REFERENCES}

1. Reibel, D. K., and Rovetto, M. J. Myocardial ATP synthesis and mechanical function following oxygen deficiency. Am. J. Physiol. 234: H247, 1978.

2. Taegtmeyer, H., Roberts, A. F., and Raine, A. E. Energy metabolism in reperfused heart muscle: Metabolic correlates to return of function. J. Am. Coll. Cardiol. 6(4): 864, 1985.

3. Bolling, S. F., Bies, L. E., Bove, E. L., and Gallagher, K. P. Augmenting intracellular adenosine improves myocardial recovery. J. Thorac. Cardiovasc. Surg. 99(3): 469, 1990.

4. Bolling, S. F., Bies, L. E., Gallagher, K. P., and Bove, E. L. Enhanced myocardial protection with adenosine. Ann. Thorac. Surg. 47: 809, 1989.

5. Ely, S. W., Mentzer, R. M., Lasley, R. D., Lee, B. K., and Berne, R. M. Functional and metabolic evidence of enhanced myocardial tolerance to ischemia and reperfusion with adenosine. $J$. Thorac. Cardiovasc. Surg. 90: 549, 1985.

6. Humphrey, S. M., Seelye, R. N., and Phil, D. Improved functional recovery of ischemic myocardium by suppression of adenosine catabolism. J. Thorac. Cardiovasc. Surg. 84: 16, 1982.

7. Ward, H. B., St. Cyr, J. A., Alyono, D., Bianco, R. W., Kriett, J. M., and Foker, J. E. Recovery of adenine nucleotide levels after global myocardial ischemia in dogs. Surgery 96(2): 248, 1984.

8. Zimmer, H. G., Trendelenburg, C., Kammermeir, H., and Gerlach, E. De novo synthesis of myocardial adenine nucleotides in the rat. Circ. Res. 32: 635, 1973.

9. Kroll, K., Kelm, M. K. M., Burrig, K. F., and Schrader, J. Transendothelial transport and metabolism of adenosine and inosine in the intact rat aorta. Circ. Res. 6: 1147, 1989.

10. Proctor, K. G. Possible role for adenosine in local regulation of absorptive hyperemia in rat intestine. Circ. Res. 59(4): 474, 1985.

11. Bache, R. J., Dai, X. Z., Schwartz, J. S., and Homans, D. C. Role of adenosine in coronary vasodilation during exercise. Circ. Res. 62(4): $846,1988$.

12. Agarwal, R. P., Specor, T., and Parks, R. E., Jr. Tight-binding inhibitors. IV. Inhibition of adenosine deaminases by various inhibitors. Biochem. Pharmacol. 26: 359, 1977.

13. Cha, S., Agarwal, R. P., and Parks, R. E., Jr. Tight-binding inhibitors. II. Nonsteady state nature of inhibition of milk xanthine oxidase by allopurinol and alloxanthine and of human erythrocytic adenosine deaminase by coformycin. Biochem. Pharmacol. 24: 2187, 1975.

14. Agarwal, R. P., Sagar, S. M., and Parkes, R. E., Jr. Adenosine deaminase from human erythrocytes: Purification and effects of adenosine analogs. Biochem. Pharmacol. 24: 693, 1975.

15. Varv, T. C., Angelakos, F. T., and Schaffer, S. W. Relationship between adenosine nucleotide metabolism and irreversible ischemic tissue damage in isolated perfused rat heart. Circ. Res. 45: $218,1979$. 